\title{
Giant Elliptical Galaxies: Globular Clusters and UCDs
}

\author{
William E. Harris ${ }^{1}$ \\ ${ }^{1}$ Physics \& Astronomy, McMaster University, Hamilton, ON L8S 4M1 Canada \\ email: harris@physics.mcmaster.ca
}

\begin{abstract}
Explorations of the globular cluster populations in many nearby galaxies are revealing increasing connections to other dense stellar systems such as UCDs, DGTOs, and nuclear star clusters in dwarf galaxies. The nearest giant elliptical, NGC 5128, is now giving us a muchimproved delineation of the GC Fundamental Plane of structural parameters, and indicates as well that the known correlation between GC scale size and metallicity is likely to be at least partly a projection effect coupled with the different spatial distributions of the metal-poor and metal-rich clusters. New photometry of the huge cluster populations around the giant Brightest Cluster Ellipticals, which allows us to study samples of many thousands of GCs at once, are now beginning to turn up surprising examples of "sequences" of high-mass GCs leading up to the UCD regime. Lastly, new modelling of cluster formation through a specially tuned semi-analytic galaxy formation code strongly suggests that the mass-metallicity relation now known to affect the blue GC sequence can arise fairly naturally out of such models, if significant numbers of the massive GCs actually represent the remnant nuclei of stripped dwarf satellites.
\end{abstract}

Keywords. galaxies: star clusters

\section{Introduction}

Our understanding of the range of properties that dense stellar systems can display in nature has experienced several healthy extensions in the past few years. Classic globular clusters, for long regarded as sitting in splendid isolation in the "fundamental plane" of structural properties of stellar systems, are now seen as having intriguing potential connections to other systems, particularly dE galaxies, Ultra-Compact Dwarfs, and the dense nuclei of dwarf galaxies. In this paper I will discuss a set of recent observations that relate to this central issue. The overarching theme is that the links between GCs and UCD-like objects are, indeed, likely to be more intricate than we had realized.

\section{NGC 5128 and its Cluster System}

NGC 5128 (Centaurus A) is the centrally dominant giant in the Centaurus galaxy group; at a distance of just $(3.8 \pm 0.2) \mathrm{Mpc}$, it is by far the nearest easily accessible giant elliptical. For comparison, the Virgo and Fornax clusters are at least three magnitudes farther away. With NGC 5128, we have a unique opportunity to study a globular cluster system in a gE galaxy at close range; and, since this galaxy contains $\simeq 1500$ GCs (Harris et al. 2006), it gives us a far larger sample of GCs to work on than all the Local Group galaxies combined.

One immediate advantage of this system is simply that we can test, quite straightforwardly, whether or not GCs in a giant elliptical are structurally similar to those in spirals and dwarfs. At $d=3.8 \mathrm{Mpc}$, a typical GC half-light diameter of $\simeq 6 \mathrm{pc}$ corresponds to an angular size of $0.33^{\prime \prime}$, which is easily resolved by HST imaging and even 
by the best-quality imaging from the ground. With HST, it is possible to resolve core radii too for many clusters, and to perform extensive tests of the basic question whether standard King-type models apply to them as well as in the Milky Way, M31, and the other Local Group galaxies. A second major advantage is that, simply because of sheer numbers, it is possible to find many "high-end" GCs with luminosities in the range of $\omega$ Cen, M31-G1, and even above. These ultramassive GCs are especially interesting because they might give clear links to other more recently discovered types of dense stellar systems - Ultra-Compact Dwarfs (UCDs), Dwarf-Globular Transition Objects (DGTOs), and nuclear star clusters in dwarf galaxies (e.g. Phillipps et al. 2001; Hasegan et al. 2005; Evstigneeva et al. 2007, among many recent studies).

The major barrier to carrying all this out in practice is a purely observational one: the GCs in NGC 5128 need to be found one by one, and since the galaxy is at moderate galactic latitude $b=19^{\circ}$, field contamination from both foreground Milky Way stars and faint, small background galaxies is extensive. High-resolution imaging and radial velocity measurement are the main tools to use in picking out GCs from everything else. The complete catalog of the $\sim 450$ GCs known to date is summarized in Woodley et al. (2007), along with a kinematic and dynamic analysis of the entire system based on the most complete available set of radial velocities. Of these known clusters, about 150 have published structural parameters based on HST ACS and STIS imaging McLaughlin et al. (2007) and more will soon be available through a similar imaging program by Jordan et al. (2007).

A sample of the results so far is shown in Fig. 1, from McLaughlin et al. (2007). This graph displays two of the most commonly used forms of the structural "fundamental plane" for GCs (see, e.g., McLaughlin 2000 and McLaughlin \& van der Marel 2005 for the most detailed recent descriptions). The two panels plot the cluster half-light radius $R_{h}$ and central velocity dispersion $\sigma$ against cluster mass. Notice particularly the change in slope of both relations above $\simeq 2 \times 10^{6} M_{\odot}$ : we are traditionally used to thinking of GCs as having a nearly uniform scale size $R_{h} \sim 3$ pc with some scatter, but at very high masses they become progressively more extended, approaching the downward extrapolation of the FP line for E galaxies. This change in the trend is also reflected in their central velocity dispersions, which continue to increase with $\mathrm{M}$ but not as rapidly as for fainter GCs. Other objects that have been isolated recently, including dwarf-globular transition objects (DGTOs; see Hasegan et al. 2006; Evstigneeva et al. 2006) and the nuclear star clusters in dE's and bulges (pointed pentagons in Fig. 1) contribute to the scatter at the high end but fall in just the same general region.

What is perhaps equally important is that the GCs in all the major nearby galaxies (NGC 5128, M31, the Milky Way) overlap completely with each other and, as far as we can tell, accurately define just the same GC fundamental plane (see especially Barmby et al. 2007 for more comparisons). The more we learn in detail about the structures, ages, and compositions of GCs in different galaxies, the more they continue to look like universal objects that are a common thread in the early history of galaxies.

\section{Cluster Size versus Location}

We are just beginning to exploit the full potential of the rich, nearby NGC 5128 system. A new ground-based imaging survey of the system has been completed with the Magellan IMACS camera, covering a $1.2 \times 1.2$-degree field centered on NGC 5128 and with a remarkable $0.45^{\prime \prime}$ resolution (Gomez et al. 2007, in preparation). This image quality makes it almost exactly equivalent to working on the Virgo and Fornax galaxies with HST. At this resolution, $90 \%$ of the GCs in NGC 5128 are resolved as nonstellar and so the heavy 


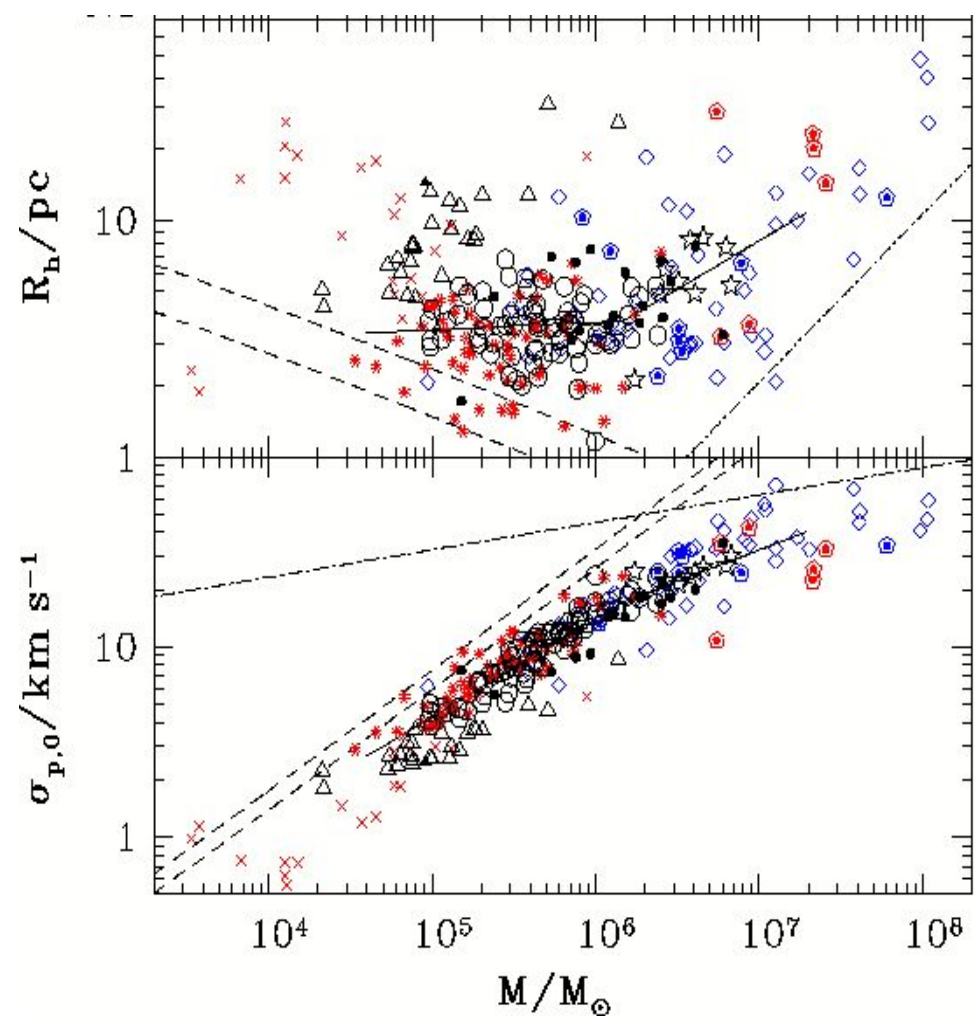

Figure 1. Two versions of the Fundamental Plane for globular clusters. The upper panel shows half-light radius versus cluster mass, the lower panel central velocity dispersion. Small crosses and stars are from the Milky Way and larger open symbols from NGC 5128. Other objects such as DGTOs and nuclear star clusters are the open pentagons with points. The pair of dashed lines in each graph show boundaries set by internal dynamical timescales: any cluster older than 20 relaxation times $\tau_{R h}$ would fall below the upper line; any older than $40 \tau_{R h}$ would fall below the lower dashed line. Most such objects would now be completely evaporated. The dot-dashed line in each graph represents a line parallel to the E-galaxy Fundamental Plane relation: E galaxies lie just above that line in the $R_{h}$ graph or just below it in the $\sigma_{p}$ graph. See Barmby et al. (2007) and McLaughlin et al. (2007) for extensive discussion.

field contamination by foreground stars is immediately removed. Furthermore, the field coverage (extending out to projected radii of $50 \mathrm{kpc}$, equivalent to about $10 R_{e f f}$ ) will allow us to explore GC properties with radius more extensively than has been done in most other systems. After further selecting objects by color and morphology, we have isolated a sample of about $1000 \mathrm{GC}$ candidates not previously known. Follow-up radial velocity programs are now underway to do the final rejection of contaminants, and will also form the basis for a much more comprehensive kinematic and dynamic study of the entire GC system.

A first scientific result from this new imaging database is a comprehensive set of measurements of structural parameters for 359 already-known clusters (Gomez \& Woodley 2007). Though the cluster core radii are too small to be resolved with anything else except HST, the half-light radii and central concentrations can be well estimated with King-type models convolved with the point spread function. Both metal-poor and metal-rich clusters display very similar global distributions in $r_{e}$, but an intriguing trend emerges when the distributions are broken down by galactocentric distance. This is shown in Fig. 2. A 
small but by-now well established correlation is for the metal-richer GCs $([\mathrm{Fe} / \mathrm{H}]>-1)$ to have systematically smaller scale radii $r_{e}$ than the metal-poorer ones $([\mathrm{Fe} / \mathrm{H}]<-1)$ in the same spatial region. This trend was first noted by Kundu \& Whitmore (1998) and Kundu et al. (1999) for NGC 3115 and M87 and was since revealed in many other systems (see particularly Larsen et al. 2001; Jordan et al. 2005 for the largest such databases). One interpretation, explored by Larsen \& Brodie (2003), is that it is a projection effect resulting from the fact that the low-metallicity clusters form a more spatially extended subsystem and thus fewer of them are deep in the central bulge region where tidal effects are strongest. This interpretation also assumes that there is a basic relation between GC size and galactocentric distance, such as the $r_{e} \sim R_{g c}^{0.5}$ scaling that applies to the Milky Way, and that both types of clusters obey at least approximately. A competing interpretation (Jordan 2004) is that it is a result strictly internal to the clusters, whereby mass segregation of their stars, coupled with shorter lifetimes on the red-giant branch for higher-metallicity stars, leads to the metal-richer clusters having smaller half-light radii.

One effective test of these two scenarios is suggested by Jordan and Larsen \& Brodie: if it is due to a projection effect within the galaxy halo, then the size differences between the metallicity groups should die away at larger $R_{g c}$, far out from the central bulge. A hint that the differences do indeed die away at large $R_{g c}$ was found by Spitler et al. (2006) for NGC 4594, where the GC measurements extend out to $\simeq 5 R_{\text {eff }}$ (see their Fig.20). However, an even stronger test can already be made within NGC 5128. As Fig. 2 indicates, a clear trend for $r_{e}$ to shrink with increasing metallicity is present for clusters projected within $1.0 R_{e f f}$; that is, within the bulge. The same trend, but weaker, is present for the next zone $1-2 R_{e f f}$. Beyond $2 R_{e f f}$, however, the correlation disappears. The only remaining effect is for increasing cluster-to-cluster scatter in their scale sizes in the outermost zones, out to $8 R_{e f f}$ and the limits of the current data (Gomez \& Woodley 2007).

This overall picture is at least roughly consistent with the Larsen/Brodie model. The reason why the previously available data did not give a clearer picture before this is simply that most of it relied on HST WFPC2 and ACS imaging of galaxies in Virgo and comparable distances, and the small field sizes of these cameras restricted the measurements to clusters within $R_{g c} \lesssim 2 R_{e f f}-$ a region clearly too restricted to see the full correlation. Once the full sample of IMAC data has been assessed, we will be able to more than double the GC sample size and confront them with more detailed modelling. One puzzle, for example, is the basic question why this trend with $R_{g c}$ should affect the half-mass radii of the clusters, which are relatively immune to dynamical evolution within the cluster, quite unlike the tidal radius. How does the cluster's location within the bulge or halo affect its intrinsic scale size? If it is not easily driven by external tidal influences during its lifetime, then once again, we may be forced to invoke the conditions at time of formation: deeper within the tidal field, the protocluster gas clouds should have already been subject to higher external pressure and thus take on smaller scale radii right from the start (Harris \& Pudritz 1994). But these descriptive ideas need to be developed much more quantitatively.

\section{Extensions to the UCD Regime: More Connections}

Connections between the "top end" of the normal GC sequence and the still higher mass range typically occupied by structures like nuclear star clusters and Ultra-Compact Dwarfs may be far more direct than even the combined data from nearby galaxies has suggested. Fig. 3 shows the luminosities and colors for a population of several thousand GCs around the supergiant elliptical NGC 3311 (the central cD galaxy in A1060). This 

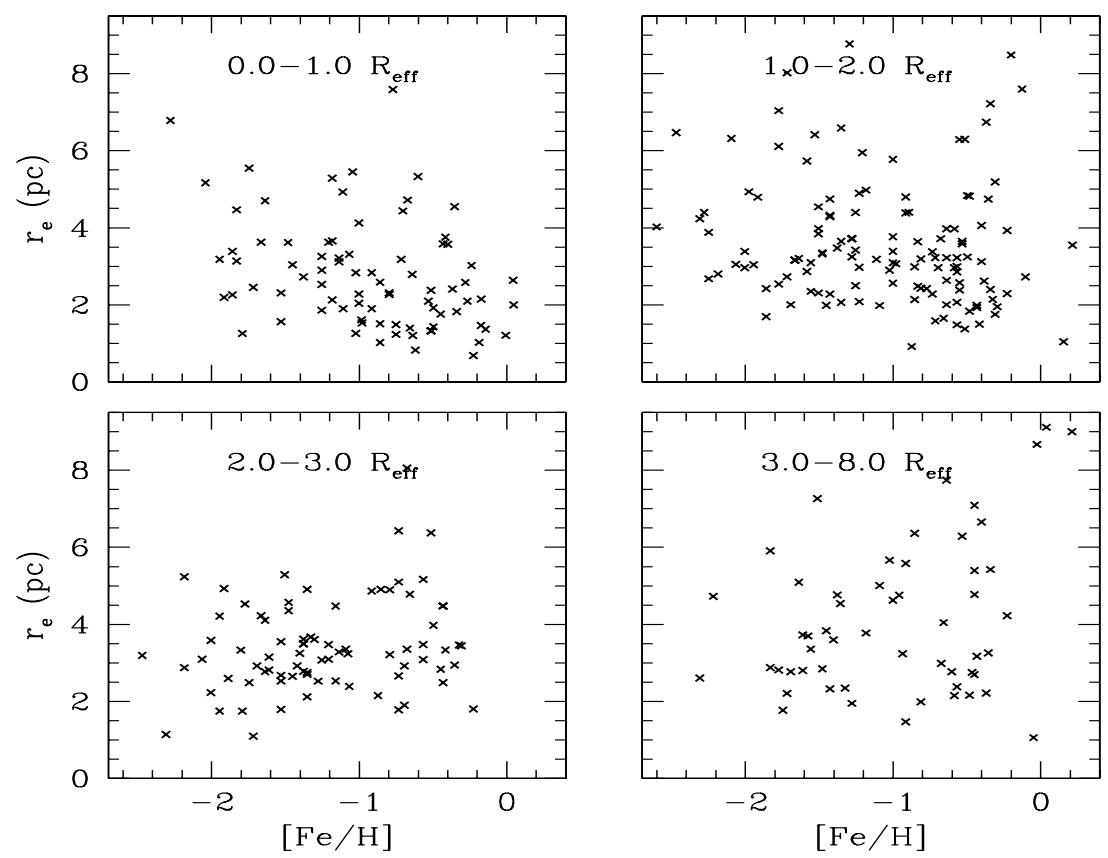

Figure 2. Effective radius $r_{e}$ (equivalent to the half-light radius) for globular clusters in NGC 5128, plotted against cluster metallicity. Four zones are shown, in steps of increasing projected galactocentric distance (in units of the galaxy effective radius). For the innermost zones, GC size decreases with increasing metallicity; for the halo beyond $\simeq 2 R_{e f f}$, no trend with metallicity is evident.

photometry (Wehner \& Harris 2007) reveals the normal bimodal color/metallicity distribution found almost everywhere, but it also shows a distinct upward extension of the red, metal-richer sequence consisting of about two dozen GCs. Archival HST/WFPC2 imaging available for about half of these shows that they are just barely more extended than the PSF width, which at the 54-Mpc distance of NGC 3311, suggests that they have typical radii $r_{e} \sim 10 \mathrm{pc}$, rather similar to UCDs and the very biggest known GCs. Their masses, assuming $M / L \simeq 3$, range from $6 \times 10^{6} M_{\odot}$ up to $3 \times 10^{7} M_{\odot}$ and thus reach into the UCD regime as well.

It is noteworthy that this type of clearly delineated sequence extension has not been seen even in composite samples of thousands of GCs resulting from combining many galaxies, either Brightest Cluster Galaxies (Harris et al. 2006) or the entire Virgo Cluster Survey (Mieske et al. 2006). Clearly there are still individual site-to-site differences in the events forming these dense, luminous stellar systems that we do not yet understand.

The blue, metal-poor sequence of GCs has gained new attention recently because it has been found to exhibit a mass-metallicity relation (MMR) whereby the more massive GCs become systematically more metal-rich, following a scaling of heavy-element abundance with mass $Z \sim M_{G C}^{0.5}$. The red GC sequence displays no such trend, staying resolutely vertical in the color-magnitude diagram. The effect was first found through metallicitysensitive color indices in several giant cD-type galaxies (Harris et al. 2006; Strader et al. 2006) and then in other galaxies of a wider range of sizes and types (Spitler et al. 2006; 


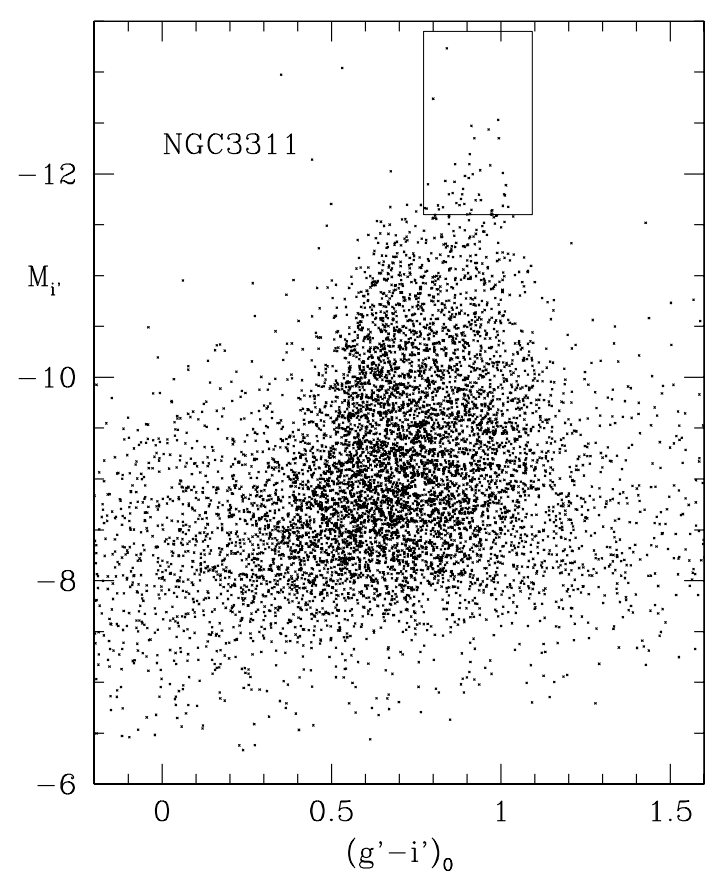

Figure 3. Luminosity versus color index for the globular cluster population around NGC 3311, the central giant elliptical in A1060 (the Hydra cluster). Note the normal "blue" and "red" GC sequences partially overlapping, but the top of the red sequence (boxed) continues upward to higher luminosity connecting with the UCD and DGTO regime.

Mieske et al. 2006). What is the origin of the MMR? Harris et al. (2006) adopt the view that the blue GCs formed at a very early stage of hierarchical merging, when they were still located within reasonably distinct pregalactic dwarfs of near-primordial gas. We then propose that on average, the more massive GCs should have formed within proportionately more massive host dwarfs (see Harris \& Pudritz 1994) that would have been more able to hold onto SNe and stellar wind ejecta and thus self-enrich, leaving behind more enriched GCs. Mieske et al. (2006) discuss several other options but favor a similar route.

This intriguing new correlation may well be giving us an entirely new window onto the properties of the pregalactic dwarfs. Quantitative models are now starting to emerge. Rothberg et al. (2007) apply the Somerville semi-analytic galaxy formation code with modifications to track the properties of the remaining dwarf galaxies within the halo of a giant galaxy, and in particular the stripped nuclei of these dwarfs. An encouraging result is that, with the same "standard" set of input model parameters found to reproduce the global properties of large galaxies, these residual nuclei fall along the upper end of the blue GC sequence and have a MMR with a realistic slope: the more massive nuclei came from systematically larger dwarfs and experienced more self-enrichment. Increases in the parameter representing the supernova heating efficiency can reduce or eliminate the MMR, while decreases can, surprisingly, turn the residual nuclei into UCD-like objects. 
Models like this clearly represent only a first tentative step into realistic interpretation. Nevertheless, the general direction looks promising, and is further reinforcing the links between these various types of compact, massive stellar systems.

\section{Acknowledgements}

The author gratefully acknowledges financial support from NSERC of Canada.

\section{References}

Barmby, P., McLaughlin, D. E., Harris, W. E., Harris, G. L. H., \& Forbes, D. A. 2007, AJ, 133, 2764

Evstigneeva, E. A., Gregg, M. D., Drinkwater, M. J., \& Hilker, M. 2007, AJ, 133, 1722

Gomez, M., \& Woodley, K. A. 2007, ApJL, submitted

Harris, W. E., Harris, G. L. H., Barmby, P., McLaughlin, D. E., \& Forbes, D. A. 2006, AJ, 132, 2187

Harris, W. E., \& Pudritz, R. E. 1994, ApJ, 429, 177

Hasegan, M. et al. 2005, ApJ, 627, 203

Jordan, A. 2004, ApJL, 613, 117

Jordan, A. et al. 2005, ApJ, 634, 1002

Jordan, A. et al. 2007, ApJL, submitted

Kundu, A., \& Whitmore, B. C. 1998, AJ, 116, 2841

Kundu, A., Whitmore, B. C., Sparks, W. B., Macchetto, F. D., Zepf, S. E., \& Ashman, K. M. 1999, ApJ, 513, 733

Larsen, S. S., \& Brodie, J. P. 2003, ApJ, 593, 618

Larsen, S. S., Brodie, J. P., Huchra, J. P., Forbes, D. A., \& Grillmair, C. J. 2001, AJ, 121, 2974

McLaughlin, D. E. 2000, ApJ, 539, 618

McLaughlin, D. E., Barmby, P., Harris, W. E., Harris, G. L. H., \& Forbes, D. A. 2007, MNRAS, submitted

McLaughlin, D. E., \& van der Marel, R. P. 2005, ApJS, 161, 304

Mieske, S. et al. 2006, ApJ, 653, 193

Phillipps, S., Drinkwater, M. J., Gregg, M. D., \& Jones, J B. 2001, ApJ, 560, 201

Rothberg, B., Harris, W. E., Somerville, R. S., Whitmore, B. C., \& Woodley, K. A. 2007, ApJL, submitted

Spitler, L. R., Larsen, S. S., Strader, J., Brodie, J. P., Forbes, D. A., \& Beasley, M. A. 2006, AJ, 132, 1593

Strader, J., Brodie, J. P., Spitler, L., \& Beasley, M. A. 2006, AJ, 132, 2333

Wehner, E. M. H., \& Harris, W. E. 2007, ApJL, in press

Woodley, K. A., Harris, W. E., Beasley, M. A., Peng, E. W., Bridges, T. J., Forbes, D. A., \& Harris, G. L. H. 2007, AJ, 134, 494 